\title{
Tendencia de la incidencia y la mortalidad en melanoma maligno en los últimos 60 años y análisis de los datos del Registro Poblacional (sic.) de Cáncer de Cali
}

Trends in cutaneous melanoma incidence and mortality in the last 60 years and analysis of the Registro Poblacional de Cáncer of Cali

\author{
Catalina Oliveros ${ }^{1}$, Juan Carlos Bravo², Ángela Zambrano³, Magda Cepeda4, Ana Francisca Ramírez ${ }^{5}$ \\ 1. Médica, residente de primer año de Dermatología, Universidad Icesi, Cali, Colombia \\ 2. Médico patólogo, Fundación Valle del Lili, Universidad Icesi, Cali, Colombia \\ 3. Médica oncóloga, Fundación Valle del Lili, Universidad Icesi, Cali, Colombia \\ 4. Médica, candidata a M.Sc. en Epidemiología, Fundación Valle del Lili, Cali, Colombia \\ 5. Médica dermatóloga, oncóloga, Fundación Valle del Lili, Universidad Icesi, Cali, Colombia
}

\section{Resumen}

Introducción. El melanoma maligno cutáneo es uno de los cánceres más agresivos que existen y uno de los de mayor crecimiento global en el mundo. La velocidad con que creció su incidencia después de 1950 ha sido tal, que ha sido considerado por muchos autores como una epidemia que azota a algunos países.

Materiales y métodos. Se hizo una búsqueda en Pubmed utilizando los términos MeSH melanoma [AND] trends; de los artículos resultantes, se utilizaron los trabajos originales, teniendo en cuenta para la discusión la incidencia, prevalencia, mortalidad, factores de riesgo, factores socioeconómicos, área corporal, sexo y edad.

Resultados y conclusiones. La incidencia del melanoma maligno cutáneo ha aumentado en la mayoría de países. El mayor número de casos y la mayor mortalidad continúan presentándose en hombres, así como las lesiones más avanzadas y de peor pronóstico, en etnias minoritarias y en personas con bajo estrato socioeconómico y pobre acceso a servicios de salud. La mortalidad ha aumentado en la población mayor de 65 años. En Cali, según el análisis del registro de cáncer de Cali, se observa que la incidencia y la mortalidad son más elevadas en hombres y mujeres mayores, con un aumento en la proporción de hombres sobre mujeres.

Palabras Clave: melanoma, tendencia, incidencia, tasa de mortalidad, prevalencia.

\section{Summary}

Background: Cutaneous melanoma is one of the most aggressive neoplasms; the number of cases has been increasing all over the world since 1950, reaching epidemic proportions.

Methods: We searched Pubmed using the MeSH terms "melanoma (AND) trends", to analyze incidence, prevalence, surveillance, risk factors, socioeconomic status, gender, localization and age of patients with melanoma.

Results and conclusions: Melanoma incidence has increased worldwide for

\author{
Correspondencia: \\ Ana Francisca Ramírez \\ Email: \\ anafranciscar@gmail.com \\ Recibido: 27 de abril de 2012. \\ Aceptado: 25 de junio de 2012.
}

No se reportan conflictos de intereses. 
men and women. The incidence and mortality is greater in males, who have thicker tumors when diagnosed; low socioeconomic status is also related to thicker tumors. Mortality and late stage melanomas are higher among adults over 65 years of age. In Cali, incidence and mortality are greater among older adults, principally in men.

KEY WORDS: Melanoma, trends, mortality rate, incidence, prevalence.

\section{Introducción}

El melanoma maligno cutáneo es uno de los cánceres con más rápido aumento y de mayor agresividad en el mundo. Se evidencia una tendencia mundial al aumento de su incidencia (estimada en un millón de casos anuales en Estados Unidos) y la estabilización de las tasas de mortalidad ${ }^{1}$. La exposición intermitente a los rayos ultravioleta es el principal factor de riesgo del melanoma maligno cutáneo ${ }^{2}$. La mayor incidencia y mortalidad continúan presentándose en hombres ${ }^{3-5}$, pero, debido a los cambios en los estilos de vida, la incidencia en mujeres ha aumentado en las últimas tres décadas ${ }^{6}$. El incremento importante que ha tenido esta enfermedad en el mundo ha llevado a que se considere una epidemia ${ }^{7,8}$.

A pesar de los esfuerzos que desde la salud pública se han generado para su prevención, al implementar campañas de educación, promoción y prevención, el problema continúa creciendo en la población general. En Colombia, aunque los fototipos de riesgo no son muy prevalentes, se ha observado una tendencia similar a la mundial en términos de prevalencia y mortalidad del melanoma maligno cutáneo?.

El objetivo de esta revisión sistemática fue examinar la literatura científica publicada sobre las tendencias de la incidencia y la prevalencia en el mundo, en términos de la evolución de la enfermedad en el tiempo y los instrumentos empleados para determinar su comportamiento en los últimos 60 años.

\section{Materiales y métodos}

\section{Revisión sistemática}

Se hizo una búsqueda sistemática de los artículos publicados en Pubmed usando los términos MeSH melanoma [AND] trends. Los artículos se seleccionaron según la pertinencia del título y del resumen, y aquellos que se relacionaran con la pregunta de investigación fueron leídos por dos revisores, quienes extrajeron la información relevante del artículo.
Del total de artículos seleccionados en la búsqueda inicial, por la revisión del título y del resumen, se descartaron los que no mostraban relación directa con el tema, los que tenían un enfoque superficial o los que no eran relevantes sobre el tema. Asimismo, se descartaron las revisiones de tema y las cartas al editor. Aquellos en los que no hubiera consenso en su inclusión, fueron resueltos con la opinión de un tercer revisor.

De los artículos resultantes, se utilizaron los trabajos originales, teniendo en cuenta para la discusión la incidencia, prevalencia, mortalidad, factores de riesgo, factores socioeconómicos, área corporal, sexo y edad. Las revisiones de tema se tuvieron en cuenta de manera informativa para el marco teórico, pero no, para la discusión y las conclusiones.

\section{Análisis secundario de registro del melanoma maligno cutáneo en Cali}

Se tomaron los datos del Registro Poblacional (sic.) de Cáncer de Cali, como base para el análisis del comportamiento nacional del melanoma maligno cutáneo. Este registro general de cáncer cuenta con datos estadísticos ininterrumpidos de los diferentes tipos de cáncer en la población de la región, desde 1962 hasta 2007. Los resultados de las tasas de mortalidad contenidas en este registro, no están discriminados por tipo de cáncer cutáneo sino como el total de las muertes por cáncer cutáneo; a pesar de ello, se tuvieron en cuenta los datos y se consideraron relevantes debido a que la mortalidad por cáncer cutáneo es atribuida globalmente, en más del $87 \%$ de los casos, al melanoma maligno.

\section{Resultados de la búsqueda}

\section{Tendencia mundial}

El melanoma maligno cutáneo es de relevancia en salud pública. Debido a que este tipo de cáncer está en un aumento continuo, se considera una epidemia a nivel mundial ${ }^{4,10}$. El melanoma maligno cutáneo constituye el $1,2 \%$ de todos los casos nuevos de cáncer en el mundo, 
con 700.00o casos nuevos diagnosticados al año, aproximadamente ${ }^{11}$. Según los datos publicados por la International Agency for Research on Cancer (IARC) en Cancer Incidence in Five Continents, durante el periodo 1978-2002, al analizar la tendencia del melanoma cutáneo a nivel mundial se observa un aumento progresivo de su incidencia máxima en esos 25 años, con un pico entre 1987 y 2002 que generó una alerta mundial por considerarse una “epidemia de melanoma” 3,4,7,12,13.

\section{Distribución mundial por países y sexo}

Es el cuarto cáncer más común en Australia y Nueva Zelanda, el décimo en Norteamérica y la península escandinava, y el decimoctavo en Gran Bretaña y Bélgica ${ }^{11}$.

El aumento en las tasas de incidencia anual de melanoma maligno cutáneo observado en las últimas seis décadas en la población (primordialmente blanca), ha sido de 3 a $7 \%$ \%,11,14; en Estados Unidos, pasó de 6 casos por 100.000 habitantes, en los años setenta, a 18, al inicio del 2000. En Canadá hubo entre 11,1 y 15,9 casos en hombres, y entre 9,8 y 14,1 casos en mujeres, por 100,000 habitantes, en los últimos 20 años ${ }^{15}$.

Esta tendencia se observó también en Europa central, donde se pasó de 304 casos por 100.000 habitantes a 10 a 15 casos, en los últimos 15 años ${ }^{16,17}$. En Gran Bretaña, se observó un aumento de la incidencia de 3,1 a 8,4 casos en hombres, y de 4,9 a 12 casos en mujeres, por 100.000 habitantes, en el periodo comprendido entre $1984 \mathrm{y}$ $2006^{2,5}$. En la cuenca del Mediterráneo, se observó un aumento de 30,94 \% en la incidencia de melanomas in situ en pacientes evaluados entre 1988 y $2006(18,19)$. En Alemania, entre 1976 y 2003, se evidenció que la incidencia de melanoma maligno cutáneo se triplicó, llegando a 10,3 casos en mujeres y a 13,3 casos en hombres, por 100.00o habitantes y por año ${ }^{12}$.

En Puerto Rico, la incidencia de melanoma maligno cutáneo aumentó un 50 \% entre 1987 y 2002, con una razón hombre a mujer de 1:1 ${ }^{20}$. El aumento en la incidencia de casos en Oceanía es el mayor de los registrados en el mundo, siendo de 40 a 60 casos por 100.000 habitantes ${ }^{14}$. La tasa más alta para ambos sexos se alcanza en Queensland (Australia), de 41,1 por 100.000 mujeres y de 55,8 por 100.000 hombres; a pesar de contar con una disminución de la incidencia en la población joven, sigue siendo muy alta en adultos mayores ${ }^{21-23}$. En Europa, la tasa más alta para ambos sexos se presentó en Suiza, con una tasa estandarizada de 19,6 por 100.000 en mujeres y de 18,6 por 100.000 en hombres. Noruega y Suiza son los países europeos que presentan las mayores incidencias de melanoma cutáneo 3,4,24.

Un estudio en los países nórdicos, de 1964 a 2003, encontró que la incidencia de melanoma maligno cutáneo se había incrementado cuatro veces durante dicho periodo, aunque fue diferente en los cinco países participantes (Noruega, Finlandia, Dinamarca, Islandia, Suecia); en el período de 1999 a 2003, dio como resultado una incidencia que oscilaba entre 9 (Finlandia) y 14,5 (Noruega) hombres por 100.000 habitantes, y entre 7,8 (Finlandia) y 19,8 (Islandia) mujeres por 100.000 habitantes. Hasta el final de la década de 1980, Islandia y Finlandia tuvieron la menor incidencia entre ambos sexos, pero, desde entonces se observó un rápido incremento en las tasas de Islandia. Los hombres y mujeres noruegos tuvieron las mayores tasas de incidencia en todo el período. Las mujeres de Islandia presentaron la incidencia más alta en los últimos cuatro años del estudio.

La mortalidad fue estable en el tiempo, contrario a la incidencia; sin embargo, hubo una duplicación de las tasas durante el período de observación, con el mayor incremento entre los hombres islandeses y noruegos. La tasa de mortalidad fue mayor entre los hombres de Noruega durante el período de estudio, mientras que los hombres finlandeses e islandeses presentaron las tasas más bajas. En el año 2003, las tasas de mortalidad variaron de 2,0 (Islandia) a 3,9 (Noruega) hombres por 100.00o habitantes, y de 1,o (Finlandia) a 2,5 (Noruega) mujeres por 100.000 habitantes. A pesar de que la incidencia fue similar en hombres y mujeres de todo el período, para todas las edades la mortalidad fue mayor entre los hombres.

La supervivencia aumentó en Noruega entre 1999 y 2003, oscilando entre 78 y $93 \%$ entre los hombres y entre 88 y $92 \%$ entre las mujeres. Los pacientes suecos siempre han tenido las mayores tasas de supervivencia, mientras que los pacientes finlandeses y daneses presentaron la menor supervivencia. El exceso de muertes por 100 personas por año fue consistentemente más alto en los hombres que las mujeres, alrededor de 2 a 5 años después del diagnóstico entre las personas mayores de 50 años. La supervivencia a cinco años fue mayor en el grupo más joven y disminuyó con la edad en todos los países a lo largo del período ${ }^{25,26}$.

El aumento de la incidencia de melanoma se observó en ambos sexos y los mayores aumentos se registraron para el melanoma del tronco, en los hombres, y para el de las extremidades, en las mujeres ${ }^{10,13,27,28}$ que siguen siendo las zonas corporales prevalentes en cada sexo. Los melanomas de cabeza y cuello han aumentado su incidencia global ${ }^{29}$. La incidencia de melanoma sigue siendo mayor en personas mayores de 50 años $^{30}$. Este comportamiento se mantuvo para Europa, Norteamérica y Suramérica ${ }^{11,19,20,31}$.

La incidencia de melanoma maligno cutáneo global no tiene un comportamiento homogéneo, lo cual se evi- 


\section{"Aunque se ha visto un aumento en la incidencia y prevalencia del melanoma maligno cutáneo en muchos países, también debe tenerse en cuenta que actualmente se diagnostica en forma más acertada y precoz, lo que podría generar un efecto de artefacto".}

dencia en los reportes europeos que muestran una incidencia mayor en mujeres (19,4 para mujeres y 14,4 para hombres, por 100.000 habitantes) $)^{7,26,32}$, en contraste con los reportes de Norteamérica y Australia, donde es mayor en hombres $(38,5$ para hombres contra 26,5 para mujeres, por 100.000 habitantes) ${ }^{3}$. En los países bajos se observa un aumento en la incidencia de melanoma maligno cutáneo, muy similar entre ambos sexos: 4,8\% en hombres y 4,3\% en mujeres, entre 1980 y $2002^{32}$.

En muchas poblaciones, el riesgo de melanoma se ha incrementado a lo largo del tiempo, siendo de un caso en 1.500, en 1935, a uno en 75, en el año $2000^{11}$. El melanoma maligno cutáneo es el cáncer de mayor incremento en la población blanca ${ }^{14}$, pero este comportamiento no es similar en grupos como los africanos, asiáticos y latinoamericanos, en los cuales la incidencia es menor que la registrada en la población blanca norteamericana, europea y de Oceanía. Además, en estos grupos de población hay una tendencia al desarrollo de lesiones en ubicaciones atípicas (protegidas del sol), de tipo distal (acral), subungular y en mucosas. Infortunadamente, este tipo de presentación poco evidente hace que el diagnóstico sea tardío ${ }^{33}$.

El gradiente de latitud se visualiza como un factor determinante en la incidencia de melanoma maligno cutáneo. Esta asociación se ve claramente en un estudio noruego con datos desde 1966 hasta 2007, donde la incidencia es 2 a 2,5 veces mayor en el sur del país; asimismo, en el sur del país, se observa una mayor incidencia de melanomas en el tronco, por la mayor exposición intermitente a los rayos ultravioleta, y en el norte, mayor incidencia en cabeza y cuello ${ }^{27}$.

La incidencia varía en Europa, donde es mayor en la península escandinava y en los países bajos que en el resto del continente, al igual que en la población australiana descendiente de inmigrantes europeos; esto se asocia con el fenotipo de la población, probablemente, con la mutación germinal en el gen CDKN2A (gen importante en el control de entrada en el ciclo celular) que se ha encontrado en determinadas zonas geográficas (Mediterráneo, Suecia y Escocia), con el pelo rojo y con las pecas. También se ha encontrado que variantes genéticas del receptor 1 de melanocortina (M1CR) se asocian con piel blanca, ojos claros, pelo rojo y una mayor predisposición a presentar un melanoma maligno. Según lo anterior, una explicación genética molecular y de factores fenotípicos evidencia la asociación entre melanoma, piel blanca y cabello rubio o rojo ${ }^{3,32}$.

Aunque se ha visto un aumento en la incidencia y prevalencia del melanoma maligno cutáneo en muchos países, también debe tenerse en cuenta que actualmente se diagnostica en forma más acertada y precoz, lo que podría generar un efecto de artefacto. No obstante, al analizar las estadísticas mundiales también se observa un subreporte de casos que puede estimarse en 9 a $25 \%$, sobre todo para tumores in situ en comparación con lesiones de mayor grosor ${ }^{11}$. Además, debido a que la mayor parte de la información se obtiene de los registros de cáncer de cada nación y estos suelen separar el melanoma lentiginoso maligno y el lentigo maligno nodular y las variedades superficiales, se genera una variación en el registro general del melanoma maligno cutáneo. No existe una política general para registrar el grosor preciso de los tumores y su localización, y se suele generar un excesivo registro de tumores secundarios o subsecuentes del mismo tipo patológico ${ }^{3}$. En Canadá, Estados Unidos y Gran Bretaña se ha demostrado que la incidencia de melanoma maligno cutáneo sí ha aumentado y no se trata únicamente de un efecto generado por el aumento en el diagnóstico precoz de la enfermedad ${ }^{34-36}$.

En el momento del diagnóstico, los melanomas presentes en mujeres suelen ser más superficiales (grosor de Breslow menor de $1 \mathrm{~mm}$ ) y, por ende, tienen mejor 
pronóstico ${ }^{37}$ que en hombres, en quienes suelen encontrarse formas más profundas ${ }^{34}$. Esto se relaciona con que los hombres se demoran más en consultar al dermatólogo y llegan con la enfermedad en estadios más avanzados ${ }^{32}$.

\section{Melanoma maligno cutáneo y diferencias étnicas}

Las minorías étnicas suelen presentar lesiones más gruesas y ulceradas, y con evidencia de compromiso sistémico ${ }^{33}$. Aún existe mucho desconocimiento sobre la etiología del melanoma en minorías étnicas con un estilo de vida, ocupación o riesgo ambiental no determinados. Aunque se sabe que la luz ultravioleta juega un papel importante en el desarrollo del melanoma maligno cutáneo, esta predisposición puede ser mayor en etnias minoritarias con ancestros europeos. Por lo anterior, las diferencias ancestrales de los grupos étnicos minoritarios tienen una significancia muy importante y generan confusión desde el punto de vista etiológico ${ }^{14}$. Lo que se encuentra es que, debido a la baja sospecha, el bajo nivel socioeconómico y el pobre acceso a los servicios de salud de los pacientes latinos y negros en los Estados Unidos, el diagnóstico de melanoma es tardío y se hace en estadios avanzados que tienen un peor pronóstico ${ }^{36,38-41}$.

En el norte y occidente de Europa, se evidencian diferencias histológicas más favorables con respecto al sur y el oriente, y estas diferencias se ven claramente afectadas por las campañas de promoción y diagnóstico temprano del melanoma en países como Noruega, Alemania y Suecia, en contraposición con países como Eslovenia, la antigua Checoslovaquia e Italia ${ }^{13}$.

\section{Factores de riesgo}

En los últimos 15 años, en Europa y Estados Unidos se ha observado un aumento de tres veces, aproximadamente, de la incidencia de melanoma en mujeres, con un aumento considerable de la presentación en el tronco de mujeres jóvenes, asociado con el cambio en el tipo de exposición, el uso de bikinis y las cámaras de bronceado $7,32,42-44$.

En Estados Unidos, el melanoma maligno cutáneo es el cáncer más común en el grupo de 25 a 29 años en ambos sexos y es el segundo cáncer más común en el grupo de mujeres con edad entre 30 y 34 años $^{11}$. La mayor esperanza de vida ha contribuido al aumento de la incidencia de melanoma en personas de edad avanzada (mayores de 60 años) y en este grupo, en especial, la enfermedad se encuentra en fase avanzada con presencia de lesiones de mayor grosor $\mathrm{y}$, por ende, de peor pronóstico ${ }^{4}$. A pesar de que el melanoma maligno cutáneo se ha incrementado por la exposición a la radiación solar, también se ha visto una disminución en la incidencia en niños y adolecentes desde los años noventa, posiblemente, secundaria a las campañas locales de salud pública ${ }^{45}$.

A pesar de existir un factor genético de predisposición para desarrollar melanoma maligno cutáneo ${ }^{46}$, la exposición intermitente a la radiación ultravioleta es el principal factor de riesgo en el desarrollo del cáncer de piel $^{2,7,47}$. La radiación ultravioleta en la superficie de la tierra contiene UVA (320-400 nm) y UVB (280-320 nm). El grupo de trabajo de la IARC clasificó como carcinógenos del grupo 1 a los rayos ultravioleta de longitud de onda entre 100 y $400 \mathrm{~nm}$ (UVA, UVB y UVC), los cuales incluyen a los emitidos por las cámaras bronceadoras ${ }^{7}$. En las últimas décadas se ha producido un cambio en los estilos de vida que conlleva a mayores exposiciones a la radiación ultravioleta. Esto, asociado a un mayor envejecimiento de la población general, lleva a tener un mayor riesgo de desarrollar cáncer de piel.

Los melanomas de tronco y extremidades están asociados con exposición a rayos ultravioleta de manera intermitente, razón por la cual están asociados a periodos vacacionales en los que se expone la piel que usualmente está cubierta en temporadas frías, mientras que la presentación en cabeza y cuello se asocia con la exposición continua a rayos ultravioleta ${ }^{42}$. Los registros muestran, globalmente, que la incidencia de melanoma maligno cutáneo ha aumentado y su presentación es más común en el tronco en los hombres y en las extremidades en las mujeres, aunque en este último grupo ha aumentado en el tronco a raíz del uso común de prendas de vestir más pequeñas. A su vez, la incidencia y prevalencia de la enfermedad continua siendo más alta en los adultos mayores. El tipo de melanoma cutáneo predominante es el superficial (grosor de Breslow de menos de $1 \mathrm{~mm}$ ), lo que se asocia a diagnósticos más tempranos de la enfermedad y ha campañas educativas de prevención. Estas características se evidencian en diferentes estudios que abarca rangos de tiempo desde 1966 hasta $2007^{5,20,27,32,48}$.

Según el estrato socioeconómico no se evidencian diferencias radicales en la incidencia de melanoma, lo cual se ha relacionado con una exposición intermitente a los rayos ultravioleta similar en todos, por contar con la misma cantidad de días de descanso o de festividades, por el uso del mismo tipo de ropa de verano y por la disminución en las tarifas de planes vacacionales que hace accesible el turismo a mayor cantidad de personas ${ }^{49}$. No obstante, sí se evidencia un aumento en la incidencia de melanoma maligno cutáneo en estadios avanzados en la población con pobre acceso a servicios de salud, debido a un bajo nivel socioeconómico ${ }^{36}$. 

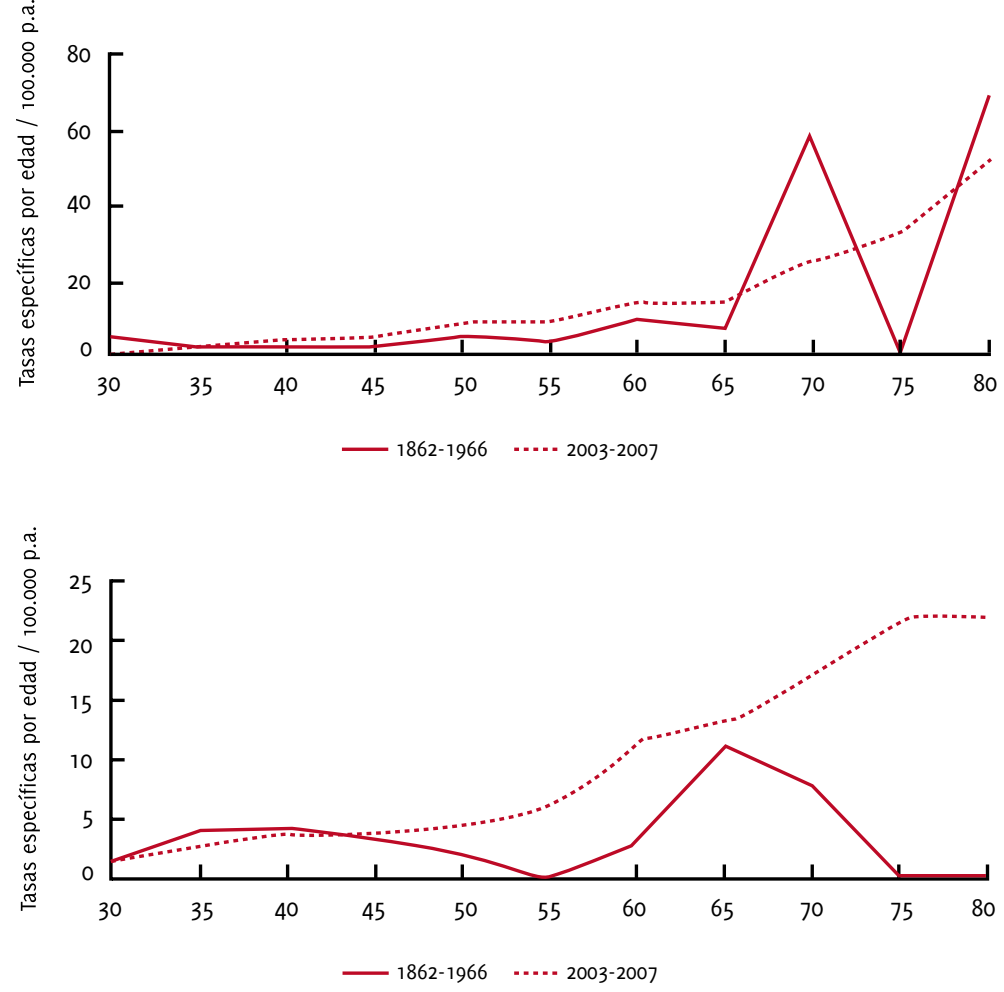

Figura 1. Tasas de incidencia de melanomas en hombres por edad, 1962-1966 y 2003-2007 Con autorización del Registro Poblacional (sic.) DE Cáncer de CaLI
Figura 2. Tasas de incidencia de melanomas en mujeres por edad, 1962-1966 y 2003-2007.

Con autorización del Registro Poblacional (sic.) DE CÁNCER DE CALI

\section{Situación del melanoma maligno cutáneo en Cali}

Con respecto a Cali, el Registro Poblacional (sic.) de Cáncer de Cali incluye el melanoma maligno cutáneo, con registro continuo desde 1962 y con datos confirmados hasta 2007, según aparece en su página web (http://rpcc.univalle.edu.co/es/index.php). Se observa que la tendencia de la incidencia de melanoma es diferente por sexo y que este cambio no ha sido igual en los periodos de 1962 a 1966 y de 2003 a 2007. Se observa que las tasas de incidencia en mujeres son constantemente más bajas comparadas con las de hombres; en ellos, la tasa llega a 20 por 100.000 hacia los 70 años, mientras que en las mujeres lo hace hacia los 75 años, en el segundo periodo observado para este evento, entre 2003 y 2007. Para el primer cuatrienio observado, entre 1962 y 1966, se encontró un pico de incidencia en hombres de 70 años, mientras que en las mujeres se había presentado de manera consistentemente mayor, pero no superaba en conjunto la observada para los hombres en este periodo (Figuras 1-3) ${ }^{9}$.

\section{Mortalidad}

La mortalidad por melanoma maligno cutáneo en diferentes países del mundo compuestos por varias razas, ha mostrado ser mayor en hombres que en mujeres y, en la raza blanca, mayor que en las otras ${ }^{4,5}$. En Gran Bretaña, las tasas de mortalidad aumentaron de o,1 a 1,7 por 100.000 personas por año para los hombres y de 0,6 a 1,8 por 100.000 personas por año para las mujeres, durante el período 1955-2007, siendo similares al inicio para ambos sexos y con un aumento en el tiempo para las mujeres hasta los años ochenta, cuando se estabilizón $0^{5,50}$.

Este tipo de cáncer presenta uno de los crecimientos de mayor ritmo a nivel mundial ${ }^{4,43}$. Se observó que para ambos sexos la tasa de mortalidad fue considerablemente diferente entre los países occidentales y los orientales, siendo mayor en los países orientales, especialmente en Australia ${ }^{8,14}$. Sin embargo, tiende a estabilizarse, sobre todo en adolescentes y adultos jóvenes, al parecer por la instauración de medidas de protección contra la radiación ultravioleta y el diagnóstico más temprano, por lo tanto, con un menor grosor de Breslow, lo que se traduce en mejor pronóstico ${ }^{4,47,51}$.

La mortalidad por melanoma aumentó desde la década de los cincuenta, con predominio en edades entre 60 y 79 años ${ }^{5,47,52}$. En el período 1960-1990, en Australia y Estados Unidos, la mortalidad en el grupo de edad de 30 a 59 años aumentó anualmente, aproximadamente, 
Figura 3.

TASAS DE INCIDENCIA DE MELANOMAS SEGÚN SEXO, 1962-2007 CON AUTORIZACIÓN DEL REGISTRO Poblacional (SIC.) de CÁNCER de CALI

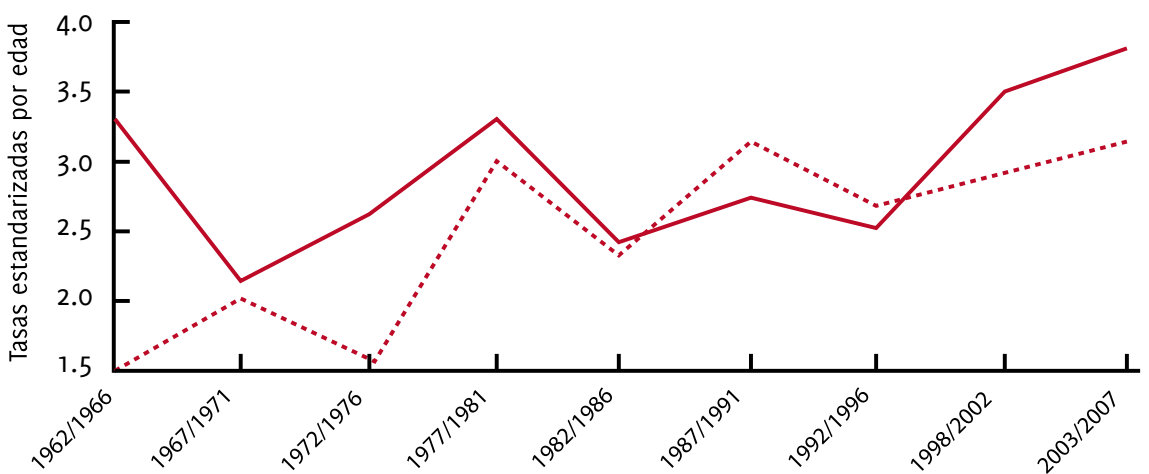

— Hombres ...... Mujeres
$2 \%$ en hombres y $1 \%$ en mujeres. El mayor aumento se encontró en Europa central, donde la tasa aumentó anualmente en 9,16\%, sin diferencias significativas según el sexo. En el grupo de edad de 60 a 79 años, los incrementos en las tasas de mortalidad fueron los mayores y predominaron en hombres ${ }^{53}$. El porcentaje anual de su aumento para hombres y mujeres de 60 a 79 años de edad fue, respectivamente, $12 \%$ y $4 \%$ en Australia, $7 \%$ y $2 \%$ en los Estados Unidos, $9 \%$ y $4 \%$ en los países nórdicos ${ }^{54,55}, 6 \%$ y $4 \%$ en Canadá, $6 \%$ y 4 \% en Japón y $7 \%$ y $5 \%$ en el Reino Unido. En el centro de Europa fue de 27 a $33 \%$ en hombres y de 15 a $24 \%$ en mujeres ${ }^{52}$.

Se puede decir que la mortalidad sufrió una estabilización global después de los años ochenta ${ }^{7,56,57}$, aunque en algunas zonas de Europa central, entre 1987 y 2003 se evidenció un aumento en la tasa de mortalidad femenina ${ }^{16}$. Esta tendencia mundial es más evidente en mujeres que en hombres ${ }^{1,44}$. En los países nórdicos se presentó, en los últimos 50 años, una disminución en la mortalidad, pero en ese tiempo se duplicó con respecto a los hallazgos previos. En los individuos más jóvenes, las tasas de mortalidad son similares en los dos sexos y están en declive ${ }^{26}$.

En Latinoamérica, específicamente en Brasil, en los últimos 25 años se evidenció un aumento en la mortalidad por melanoma maligno cutáneo, mayor en hombres que en mujeres. A pesar de la heterogeneidad étnica de la región, la mortalidad fue mayor en blancos, que se considera viven en su mayoría al sur del continente. La incidencia fue mayor en edades por encima de los 50 años, con tendencia a la estabilización en menores de esa edad ${ }^{5}$. Los reportes mundiales sugieren que hay pequeños descensos en la mortalidad por esta enfermedad, los cuales fueron evidentes inicialmente en Australia, Estados Unidos y los países nórdicos, seguidos de Gran Bretaña, Alemania y Canadá35.59. Por el contrario, en algunos países europeos centrales (Francia, Italia, República Checa y Eslovaquia), se observa que la mortalidad sigue aumentando, claro está, con pequeñas excepciones locales ${ }^{60}$. Lo anterior sugiere que las diferentes poblaciones se encuentran, al mismo tiempo, en diferentes niveles de la curva de la mortalidad por melanoma maligno cutáneo, lo que puede estar relacionado con las diferencias en la implementación de los planes de promoción y prevención en los diferentes países ${ }^{22}$, o con la inmunoterapia adyuvante o quimioinmunoterapia usada en los pacientes con enfermedad avanzada, que antes no se utilizaba ${ }^{60}$. Solo la educación sobre melanoma maligno cutáneo y la implementación de los planes de promoción y prevención, sobre todo el diagnóstico precoz de la enfermedad, generarán una disminución significativa en la mortalidad por melanoma ${ }^{57}$, ya que los factores de riesgo externos continuarán siendo inamovibles.

Con respecto al registro de Cali ${ }^{9}$, la tasa de mortalidad no muestra diferencias importantes en sus cifras en las mujeres, entre los dos periodos observados (1984-1988 y 2004-2008). Sin embargo, se observa de manera característica un descenso en la mortalidad en hombres en el primer periodo observado desde los 75 años, mientras que en mujeres muestra solo un ligero descenso. Para el segundo periodo observado, existe una tendencia similar en la mortalidad hasta los 65 años, edad a partir de la cual se acelera más la mortalidad en hombres, para alcanzar la tasa de 30 por 10.000 para los 80 años, mientras que en las mujeres apenas supera los 15 por 10.000 personas (Figuras 4-6) ${ }^{9}$.

Independientemente de la edad de los pacientes, que 

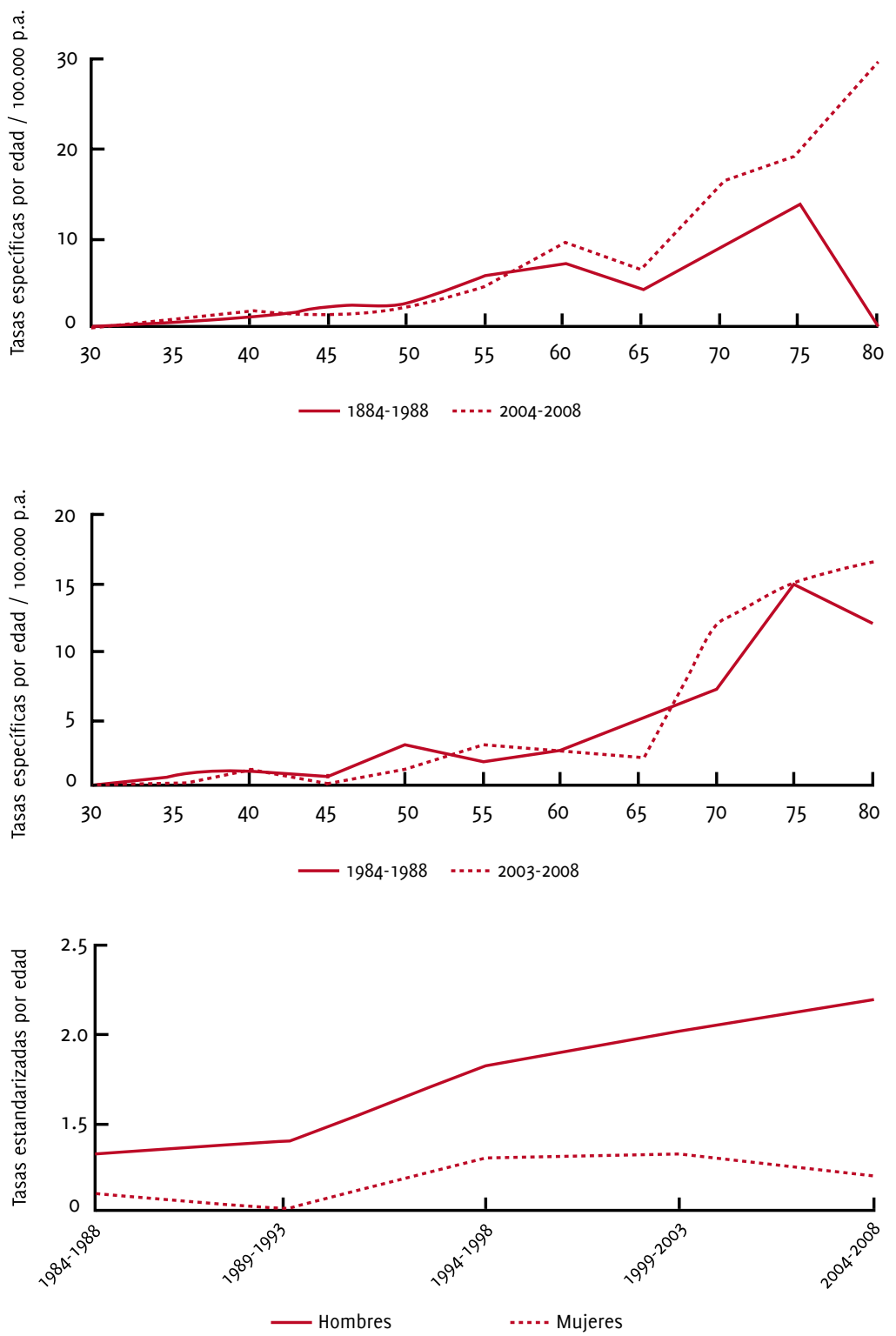

Figura 4. Tasas de mortalidad para hombres por edad, por melanoma y otros cánceres de piel, 1984-1988 y 2004-2008

Con autorización del Registro Poblacional (sic.) DE CÁNCER DE CALI
FiguRA 5. Tasas de mortalidad para mujeres por edad, por melanoma y otros cánceres de piel, 1984-1988 y 2004-2008

Con autorización del Registro Poblacional (sic.) DE CÁNCER DE CALI
Figura 6. Tasas de mortalidad por sexo por melanoma y otros cánceres de piel, 1984-2008 Con autorización del Registro Poblacional (sic.) De CÁNCER DE CALI podría explicar el efecto temporal sobre la tendencia de la incidencia, al ajustar por edad se observa que en los hombres la incidencia de melanoma es constantemente superior que en las mujeres, desde 1982 hasta 2007, con una ligera inversión de la curva entre 1982 y 1996; aunque esto no es comparable con las curvas de mortalidad, ya que estas incluyen otros tipos de cáncer de piel, se observa una tendencia significativamente superior en hombres que en mujeres a lo largo de todo el periodo de observación'. Probablemente, al igual que en los países subdesarrollados o en desarrollo, esto refleja deficiencias en el acceso a servicios médicos com- petentes, demoras en la asignación de consultas y otros problemas socioeconómicos.

La tendencia al alza en la incidencia y la supervivencia del melanoma maligno cutáneo en los países nórdicos, puede estar relacionada, principalmente, con el resultado de grandes cambios en los hábitos de tomar el sol o los rayos ultravioleta y otros, y más recientemente, con mayor conciencia en la comunidad médica y el público sobre la detección precoz del melanoma ${ }^{26}$.

Por el comportamiento que ha presentado, se prevé que su incidencia continuará aumentando, con predominio en la raza blanca; esta tendencia se mantendrá a nivel 


\section{"La mortalidad por melanoma ha tendido a estabilizarse en los últimos 30 años, gracias al diagnóstico temprano y a la mejor supervivencia que esto conlleva, pero, sigue siendo mayor en hombres que en mujeres $y$, en ambos sexos, en la sexta y la séptima décadas de vida".}

mundial, al menos en los próximos 20 años ${ }^{4}$, pudiendo llegar a un aumento de hasta $100 \%{ }^{61-63}$, excepto en la población joven, en la cual se presume que disminuiráa ${ }^{64}$. Este fenómeno se ve agravado por el daño continuo en la capa de ozono y por la cultura del bronceado ${ }^{47}$. La tendencia en la presentación histológica del melanoma maligno cutáneo, seguirá siendo una mayor incidencia de las formas superficiales (delgados, de menos de $1 \mathrm{~mm}$ ), con mejor pronóstico en cuanto a la supervivencia ${ }^{37,65}$; esto se debe a las crecientes campañas de promoción y prevención en los diferentes países, al uso de bloqueadores solares y al diagnóstico temprano

\section{Discusión}

En los últimos 60 años, la incidencia y la prevalencia del melanoma maligno cutáneo han aumentado de manera global en todo el mundo, con cifras significativas; han llegado a un aumento de $7 \%$ en algunos países, que se espera sea de $100 \%$ en los próximos 20 años.

A mediados de la década de los ochenta, el pico de incidencia llegó al máximo, lo cual coincidió con el aumento de la exposición solar vacacional, el uso de vestidos de baño más pequeños y la exposición de un área mayor de piel por actividades deportivas y las cámaras de bronceado. La incidencia ha aumentado en las mujeres jóvenes, debido al comportamiento asociado con la cultura del bronceado, lo que se evidencia de manera más notoria en poblaciones con predominio de los fototipos I y II. La incidencia en niños y adolecentes ha tendido a disminuir, gracias a las campañas de los gobiernos de algunos países que fomentan la exposición mesurada al sol. En promedio, la edad de mayor incidencia es la sexta década, tanto en hombres como en mujeres; sin embargo, según el estadio histológico, los hombres presentan enfermedad más avanzada $\mathrm{y}$, por ende, con peor pronóstico.

En la mayoría de los países, la incidencia del melanoma ha aumentado a expensas de aquellos in situ o delgados, lo que se asocia con más y mejores diagnós- ticos en los estadios iniciales, gracias a la concientización de la población y a las campañas de promoción y prevención de los gobiernos. En general, las minorías étnicas presentan menor incidencia de melanoma maligno cutáneo, pero suele diagnosticarse en estadios avanzados. Esto se asocia con diagnósticos tardíos por baja sospecha y con dificultados en el acceso a servicios de salud de calidad por deficiencias socioeconómicas.

La exposición intermitente a los rayos ultravioleta (A, B y C) continúa considerándose el principal factor de riesgo para desarrollar melanoma maligno cutáneo, incluida la radiación de las cámaras de bronceado. Las zonas corporales más afectadas continúan siendo el tronco en los hombres y los miembros inferiores en las mujeres, pero, debido a la exposición de más área corporal, la incidencia en el tronco de las mujeres está en aumento. El melanoma maligno en cabeza y cuello ha presentado un incremento relacionado con la edad del paciente.

La mortalidad por melanoma ha tendido a estabilizarse en los últimos 30 años, gracias al diagnóstico temprano y a la mejor supervivencia que esto conlleva, pero, sigue siendo mayor en hombres que en mujeres $\mathrm{y}$, en ambos sexos, en la sexta y la séptima décadas de vida. Para evitar el desarrollo de esta enfermedad, se considera absolutamente necesario implementar y mejorar las campañas de promoción y prevención, incluyendo el uso de bloqueadores solares, la exposición controlada a los rayos ultravioleta y su diagnóstico temprano ante cualquier sospecha.

\section{Referencias}

1. Jemal A, Devesa SS, Hartge P, Tucker MA. Recent trends in cutaneous melanoma incidence among whites in the United States. J Natl Cancer Inst. 2001;93:678-83.

2. Hall HI, Miller DR, Rogers JD, Bewerse B. Update on the incidence and mortality from melanoma in the United States. J Am Acad Dermatol. 1999;40:35-42.

3. MacKie RM, Hauschild A, Eggermont AM. Epidemiology of invasive cutaneous melanoma. Ann Oncol.2009;20(Suppl.6):1-7. 
4. Aceituno-Madera P, Buendía-Eisman A, Arias-Santiago S, Serrano-Ortega S. Changes in the incidence of skin cancer between 1978 and 2002. Actas Dermosifiliogr. 2010;101:39-46.

5. Montella A, Gavin A, Middleton R, Autier P, Boniol M. Cutaneous melanoma mortality starting to change: A study of trends in Northern Ireland. Eur J Cancer. 2009;45:2360-6.

6. Bulliard JL, Cox B. Cutaneous malignant melanoma in New Zealand: Trends by anatomical site, 1969-1993. Int J Epidemiol. 2000;29:416-23.

7. Héry C, Tryggvadóttir L, Sigurdsson T, Olafsdóttir E, Sigurgeirsson B, Jonasson JG, et al. A melanoma epidemic in Iceland: Possible influence of sunbed use. Am J Epidemiol. 2010;172:762-7.

8. Marcos-Gragera R, Vilar-Coromina N, Galceran J, Borràs J, Clèries $\mathrm{R}$, Ribes J, et al. Rising trends in incidence of cutaneous malignant melanoma and their future projections in Catalonia, Spain: Increasing impact or future epidemic? J Eur Acad Dermatol Venereol. 2010;24:1083-8.

9. Registro Poblacional de Cáncer de Cali, Colombia. Melanoma, 1962-2007. Fecha de consulta: 11 de agosto de 2011. Disponible en: rpcc.univalle.edu.co.

10. Glass AG, Hoover RN. The emerging epidemic of melanoma and squamous cell skin cancer. JAMA. 1989;262:2097-100.

11. Brochez L, Naeyaert JM. Understanding the trends in melanoma incidence and mortality: Where do we stand? Eur J Dermatol. 2000;10:71-5.

12. Lasithiotakis KG, Leiter U, Gorkievicz R, Eigentler T, Breuninger $\mathrm{H}$, Metzler $\mathrm{G}$, et al. The incidence and mortality of cutaneous melanoma in Southern Germany: Trends by anatomic site and pathologic characteristics, 1976 to 2003. Cancer. 2006;107:1331-9.

13. de Vries E, Bray FI, Eggermont AM, Coebergh JW; European Network of Cancer Registries. Monitoring stage-specific trends in melanoma incidence across Europe reveals the need for more complete information on diagnostic characteristics. Eur J Cancer Prev. 2004;13:387-95.

14. Garbe C, Leiter U. Melanoma epidemiology and trends. Clin Dermatol. 2009;27:3-9.

15. Metelitsa AI, Dover DC, Smylie M, de Gara CJ, Lauzon GJ. A population-based study of cutaneous melanoma in Alberta, Canada (1993-2002). J Am Acad Dermatol. 2010;62:227-32.

16. Chellini E, Crocetti E, Carli P, Martini A, Giovannetti L. The melanoma epidemic debate: Some evidence for a real phenomenon from Tuscany, Italy. Melanoma Res. 2007;17:129-30.

17. Pellacani G, Lo Scocco G, Vinceti M, Albertini G, Raccagni AA, Baldassari L, et al .Melanoma epidemic across the millennium: Time trends of cutaneous melanoma in Emilia-Romagna (Italy) from 1997 to 2004. J Eur Acad Dermatol Venereol. 2008;22:213-8.

18. Marcoval J, Moreno A, Torras A, Baumann E, Graells J, Gallego MI. Changes in incidence of malignant melanoma in the last 19 years in a tertiary hospital on the Mediterranean coast. Actas Dermosifiliogr. 2008;99:464-8.

19. Cayuela A, Rodríguez-Domínguez S, Lapetra-Peralta J, ConejoMir JS. Has mortality from malignant melanoma stopped rising in Spain? Analysis of trends between 1975 and 2001. Br J Dermatol. 2005;152:997-1000.

20. Valentín SM, Sánchez JL, Figueroa LD, Nazario CM. Epidemiology of melanoma in Puerto Rico, 1987-2002. P R Health Sci J. 2007;26:343-8.

21. Whiteman DC, Bray CA, Siskind V, Green AC, Hole DJ, Mackie RM. Changes in the incidence of cutaneous melanoma in the west of Scotland and Queensland, Australia: hope for health promotion? Eur J Cancer Prev. 2008;17:243-50.

22. Coory M, Baade P, Aitken J, Smithers M, McLeod GR, Ring I. Trends for in situ and invasive melanoma in Queensland, Australia, 1982-2002. Cancer Causes Control. 2006;17:21-7.
23. Buettner PG, MacLennan R. Geographical variation of incidence of cutaneous melanoma in Queensland. Aust J Rural Health. 2008;16:269-77.

24. Holterhues C, Vries E, Louwman MW, Koljenovi S, Nijsten T. Incidence and trends of cutaneous malignancies in the Netherlands, 1989-2005. J Invest Dermatol. 2010;130:1807-12.

25. Hallberg O. A theory and model to explain the skin melanoma epidemic. Melanoma Res. 2006;16:115-8.

26. Tryggvadóttir L, Gislum M, Hakulinen T, Klint A, Engholm G, Storm HH, et al. Trends in the survival of patients diagnosed with malignant melanoma of the skin in the Nordic countries 19642003 followed up to the end of 2006. Acta Oncol. 2010;49:665-72.

27. Cicarma E, Juzeniene A, Porojnicu AC, Bruland ØS, Moan J. Latitude gradient for melanoma incidence by anatomic site and gender in Norway 1966-2007. J Photochem Photobiol B. 2010;101:174-8.

28. Pérez-Gómez B, Aragonés N, Gustavsson P, Lope V, López-Abente G, Pollán M. Do sex and site matter? Different age distribution in melanoma of the trunk among Swedish men and women. $\mathrm{Br} \mathrm{J}$ Dermatol. 2008;158:766-72.

29. Stang A, Pukkala E, Sankila R, Söderman B, Hakulinen T. Time trend analysis of the skin melanoma incidence of Finland from 1953 through 2003 including 16,414 cases. Int J Cancer. 2006;119:380-4.

30. Newnham A, Møller H. Trends in the incidence of cutaneous malignant melanomas in the south east of England, 1960-1998. J Public Health Med. 2002;24:268-75.

31. Bulliard JL, Cox B, Semenciw R. Trends by anatomic site in the incidence of cutaneous malignant melanoma in Canada, 1969-93. Cancer Causes Control. 1999;10:407-16.

32. de Vries E, Houterman S, Janssen-Heijnen ML, Nijsten T, van de Schans SA, Eggermont AM, et al. Up-to-date survival estimates and historical trends of cutaneous malignant melanoma in the south-east of The Netherlands. Ann Oncol. 2007;18:1110-6.

33. Shoo BA, Kashani-Sabet M. Melanoma arising in African-, Asian-, Latino- and Native-American populations. Semin Cutan Med Surg. 2009;28:96-102.

34. Ulmer MJ, Tonita JM, Hull PR. Trends in invasive cutaneous melanoma in Saskatchewan 1970-1999. J Cutan Med Surg. 2003;7:433-42.

35. Downing A, Newton-Bishop JA, Forman D. Recent trends in cutaneous malignant melanoma in the Yorkshire region of England; incidence, mortality and survival in relation to stage of disease, 1993-2003. Br J Cancer. 2006;95:91-5.

36. Linos E, Swetter SM, Cockburn MG, Colditz GA, Clarke CA. Increasing burden of melanoma in the United States. J Invest Dermatol. 2009;129:1666-74.

37. Levi F, Te VC, Randimbison L, La Vecchia C. Trends in incidence of various morphologies of malignant melanoma in Vaud and Neuchatel, Switzerland. Melanoma Res. 2005;15:73-5.

38. Rouhani P, Hu S, Kirsner RS. Melanoma in Hispanic and black Americans. Cancer Control. 2008;15:248-53.

39. Rouhani P, Pinheiro PS, Sherman R, Arheart K, Fleming LE, Mackinnon J, et al. Increasing rates of melanoma among nonwhites in Florida compared with the United States. Arch Dermatol. 2010;146:741-6.

40. Hu S, Parmet Y, Allen G, Parker DF, Ma F, Rouhani P, Kirsner RS. Disparity in melanoma: A trend analysis of melanoma incidence and stage at diagnosis among whites, Hispanics, and blacks in Florida. Arch Dermatol. 2009;145:1369-74.

41. Bellows CF, Belafsky P, Fortgang IS, Beech DJ. Melanoma in African-Americans: Trends in biological behavior and clinical characteristics over two decades. J Surg Oncol. 2001;78:10-6. 
42. Bradford PT, Anderson WF, Purdue MP, Goldstein AM, Tucker MA. Rising melanoma incidence rates of the trunk among younger women in the United States. Cancer Epidemiol Biomarkers Prev. 2010;19:2401-6.

43. Dal H, Boldemann C, Lindelöf B. Does relative melanoma distribution by body site 1960-2004 reflect changes in intermittent exposure and intentional tanning in the Swedish population? Eur J Dermatol. 2007;17:428-34.

44. Nieto A, Ruiz-Ramos M, Abdel-Kader L, Conde M, Camacho F. Gender differences in rising trends in cutaneous malignant melanoma in Spain, 1975-98. Br J Dermatol. 2003;148:110-6.

45. Karlsson PM, Fredrikson M. Cutaneous malignant melanoma in children and adolescents in Sweden, 1993-2002: The increasing trend is broken. Int J Cancer. 2007;121:323-8.

46. Hemminki K, Zhang H, Czene K. Incidence trends and familial risks in invasive and in situ cutaneous melanoma by sun-exposed body sites. Int J Cancer. 2003;104:764-71.

47. Gaudette LA, Gao RN. Changing trends in melanoma incidence and mortality. Health Rep. 1998;10:29-41.

48. Lipsker D, Engel F, Cribier B, Velten M, Hedelin G. Trends in melanoma epidemiology suggest three different types of melanoma. Br J Dermatol. 2007;157:338-43.

49. Doherty VR, Brewster DH, Jensen S, Gorman D. Trends in skin cancer incidence by socioeconomic position in Scotland, 19782004. Br J Cancer. 2010;102:1661-4.

50. Streetly A, Markowe H. Changing trends in the epidemiology of malignant melanoma: Gender differences and their implications for public health. Int J Epidemiol. 1995;24:897-907.

51. Cayuela A, Rodríguez-Domínguez S, Vigil E, Conejo-Mir JS. Effect of age, birth cohort and period of death on skin melanoma mortality in Spain, 1975 through 2004. Int J Cancer. 2008;122:905-8.

52. Severi G, Giles GG, Robertson C, Boyle P, Autier P. Mortality from cutaneous melanoma: Evidence for contrasting trends between populations. Br J Cancer. 2000;82:1887-91.

53. Stang A, Valiukeviciene S, Aleknaviciene B, Kurtinaitis J. Time trends of incidence, mortality, and relative survival of invasive skin melanoma in Lithuania. Eur J Cancer. 2006;42:660-7.

54. Månsson-Brahme E, Johansson $\mathrm{H}$, Larsson $\mathrm{O}$, Rutqvist LE, Ringborg U. Trends in incidence of cutaneous malignant melanoma in a Swedish population 1976-1994. Acta Oncol. 2002;41:138-46.
55. Bleyen L, De Bacquer D, Myny K, Brochez L, Naeyaert JM, De Backer G. Trends in mortality from cutaneous malignant melanoma in Belgium. Int J Epidemiol. 1999;28:40-5.

56. Giles GG, Armstrong BK, Burton RC, Staples MP, Thursfield VJ. Has mortality from melanoma stopped rising in Australia? Analysis of trends between 1931 and 1994. BMJ. 1996;312:1121-5.

57. Baade P, Coory M. Trends in melanoma mortality in Australia: 1950-2002 and their implications for melanoma control. Aust N Z J Public Health. 2005;29:383-6.

58. Mendes GL, Koifman RJ, Koifman S. Mortality frequency and trends attributed to melanoma in Brazil from 1980-2005. J Toxicol Environ Health A. 2010;73:850-7.

59. Stang A, Jöckel KH. Changing patterns of skin melanoma mortality in West Germany from 1968 through 1999. Ann Epidemiol. 2003;13:436-42.

6o. Crocetti E, Carli P. Unexpected reduction of mortality rates from melanoma in males living in central Italy. Eur J Cancer. 2003;39:818-21.

61. Crocetti E, Carli P, Miccinesi G. Melanoma incidence in central Italy will go on increasing also in the near future: A registry-based, age-period-cohort analysis. Eur J Cancer Prev. 2007;16:50-4.

62. Zhou J, Enewold L, Zahm SH, Devesa SS, Anderson WF, Potter JF, McGlynn KA, Zhu K. Melanoma incidence rates among whites in the U.S. Military. Cancer Epidemiol Biomarkers Prev. 2011;20:318-23.

63. Klit A, Drejøe JB, Drzewiecki KT. Trends in the incidence of malignant melanoma in Denmark, 1978-2007. Incidence on the island of Bornholm compared with the whole country incidence in Denmark. Dan Med Bull. 2011;58:A4229.

64. Bosetti C, La Vecchia C, Naldi L, Lucchini F, Negri E, Levi F. Mortality from cutaneous malignant melanoma in Europe. Has the epidemic leveled off? Melanoma Res. 2004;14:301-9.

65. Balzi D, Carli P, Giannotti B, Paci E, Buiatti E. Cutaneous melanoma in the Florentine area, Italy: Incidence, survival and mortality between 1985 and 1994. Eur J Cancer Prev. 2003;12:43-8.

66. Insinga RP, Reither EN, Remington PL, Stephenson-Vine L. Trends in malignant melanoma incidence and mortality in Wisconsin, 1979-1997. WMJ. 2001;100:27-31. 